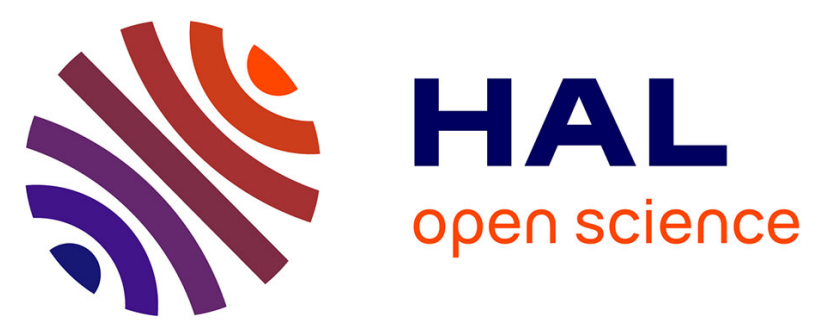

\title{
Optimized scoring tool to quantify the functional performance during the sit-to-stand transition with a magneto-inertial measurement unit
}

Kevin Lepetit, Khalil Ben Mansour, Adrien Letocart, Sofiane Boudaoud, Kiyoka Kinugawa, Jean-François Grosset, Frédéric Marin

\section{To cite this version:}

Kevin Lepetit, Khalil Ben Mansour, Adrien Letocart, Sofiane Boudaoud, Kiyoka Kinugawa, et al.. Optimized scoring tool to quantify the functional performance during the sit-to-stand transition with a magneto-inertial measurement unit. Clinical Biomechanics, 2019, 69, pp.109-114. 10.1016/j.clinbiomech.2019.07.012 . hal-02406859

\author{
HAL Id: hal-02406859 \\ https://hal.utc.fr/hal-02406859
}

Submitted on 12 Dec 2019

HAL is a multi-disciplinary open access archive for the deposit and dissemination of scientific research documents, whether they are published or not. The documents may come from teaching and research institutions in France or abroad, or from public or private research centers.
L'archive ouverte pluridisciplinaire HAL, est destinée au dépôt et à la diffusion de documents scientifiques de niveau recherche, publiés ou non, émanant des établissements d'enseignement et de recherche français ou étrangers, des laboratoires publics ou privés. 
4 Type of manuscript: Original paper

5 Authors' names and affiliations:

6
University, Dr Schweitzer Street, 60200 Compiègne, France

(2) Pitié-Salpêtrière Hospital - Charles Foix Hospital (AP-HP), Avenue de la République, 94200 Ivry-sur-Seine, France

(3) Université Paris 13, Sorbonne Paris Cité, UFR Santé Médecine et Biologie Humaine, 93017 Bobigny, France 
17 Abstract: 243 words

18 Main text: 2830 words

19

20

21

22

23

24

25

26

27

28

29

30

31

32 
Background: Sit-to-stand is used as a qualitative test to evaluate functional performance, especially to detect fall risks and frail individuals. The use of various quantitative criteria would enable a better understanding of musculoskeletal deficits and movement strategy modifications. This quantification was proven possible with a magneto-inertial unit which provides a compatible wearable device for clinical routine motion analysis.

Methods: Sit-to-stand movements were recorded using a single magneto-inertial measurement unit fixed on the chest for 74 subjects in three groups healthy young, healthy senior and frail. MIMU data was used to compute 15 spatiotemporal, kinematic and energetic parameters. Nonparametric statistical test showed a significant influence of age and frailness. After reducing the number of parameters by a principal component analysis, an AgingScore and a FrailtyScore were computed.

Findings: The fraction of variance explained by the first principal component was $77.48 \pm 2.80 \%$ for principal component analysis with healthy young and healthy senior groups, and $74.94 \pm 2.24 \%$ with healthy and frail senior groups. By receiver operating characteristic curve analysis of this score, we were able to refine the analysis to differentiate between healthy young and healthy senior subjects as well as healthy senior and frail subjects. By radar plot of the most discriminate parameters, the motion's strategy could be characterized and be used to detect premature functional deficit or frail subjects. 
Interpretation: Sit-to-stand measured by a single magneto-inertial unit and dedicated post processing

is able to quantify subject's musculoskeletal performance and will allow longitudinal investigation of aging population.

Keywords: sit-to-stand; magneto-inertial measurement unit; frailty; age; biomechanics

\section{INTRODUCTION}

The sit-to-stand (STS) movement is one of the most commonly performed daily tasks (Nuzik et al., 1986). This postural transition requires coordination, balance, strength and muscle power (Millor et al., 2014) which become difficult with age (Alexander et al., 1991). Mobility is reduced with age due to illness, trauma, or progressive deconditioning i.e. sarcopenia, osteoporosis (Millor et al., 2014). The STS transition is often used to monitor the seniors and evaluate physical performance (Mijnarends et al., 2013). In practice, the clinical evaluation of the STS is based on motion description to investigate motor strategy modification (Millington et al., 1992). As quantification, the task duration is classically used as a descriptor of the STS transition performance (Beauchet et al., 2011; Millor et al., 2014). However this parameter is global, and not specific enough to quantify deficit in seniors (Lepetit et al. 2018).

The recent development of wearable magneto-inertial measurement unit (MIMU) has led to new opportunities for clinical assessment of STS performance (Howcroft et al., 2013; Lepetit et al., 2018; Millor et al., 2013; Sun \& Sosnoff, 2018) with the advantage to be intended for clinical routine use 

diagnostic frailty (Mugueta-Aguinaga \& Garcia-Zapirain, 2017) or estimate fall risks (Howcroft et al., 2013; Sun \& Sosnoff, 2018). However, these studies focused on populations with diagnosed pathologies. In aging populations with risk for sarcopenia, the loss of tonicity or sedentariness should be monitored early to detect the firsts signs indicating a significant weakness of the subject (CruzJentoft et al. 2019).

The use of a MIMU during the STS has be demonstrated to be relevant (Millor et al., 2014) and results showed an increase of task duration and a decrease of flexion angular velocity and coefficient of variations (i.e. ratio between standard deviation and mean durations) with age (R. C. Van Lummel et al., 2013). However, few parameters take into account the subject's morphology in the STS performance (Ganea et al., 2011; Zijlstra et al., 2010). In addition, it may be relevant to combine significant parameters in order to create a score that classifies individuals according to their mobility health status (Millor et al., 2014).

The aim of this study is to design a diagnostic tool to detect functional deficit based on a single MIMU during the STS. Investigations will focus on age and frailty effects on kinematic and kinetic parameters extracted from data of a MIMU fixed on the chest during the STS postural transition to deduce a functional score which enable to differentiate frail from healthy senior individuals and healthy senior from young subjects. 


\section{MATERIALS AND METHODS}

Seventy-four subjects were enrolled in this study (table 1). They were divided into three groups: healthy young (HY), healthy senior (HS) and frail senior (FS). To be eligible, healthy young subjects had to be asymptomatic between 18 and 30 years old. Healthy senior subjects had to be over 65 years old and asymptomatic after examination by a medical doctor. The study also included 11 frail senior subjects after examination of a geriatrician. Geriatricians define frailty as a biologic syndrome of decreased reserve and resistance to stressors, resulting from cumulative declines across multiple physiologic systems, and causing vulnerability to adverse outcomes (Fried et al., 2001). Frail subjects had to be over 65 years old and have a degree of frailty greater than 5 according to Rockwood index

100 (Rockwood et al., 2005).

Insert table 1

All the volunteers gave their free and written consent for these experiments. The protocol was approved by the ethical committee of Nord-Ouest II number 2016-A00534-47 and ethical committee of Ile-de-France VI in 2016.

\subsection{Instrumentation}

Participants were instrumented with a MIMU (APDM, Opal, Portland, USA) fixed, with an elastic strap, on their chest at approximately two thirds of the breastbone. The MIMU was composed of a 3D 
gyroscope, a 3D accelerometer and a 3D magnetometer. The height of the chair used for this study was standard $(45 \mathrm{~cm})$. The signals of the MIMU data were sampled at $128 \mathrm{~Hz}$.

After a static sitting pose, the subjects were asked to stand up at self-pace without assistance and

112 without using their hands. Each participant performed three to five STS transitions according to their

113 physical conditions. Each transition was recorded separately. A 1-minute rest period was done

114 between each test.

115 After the session, the weight and height of each subject were measured using a weighing scale and a 116 measuring stick.

Based on fusion algorithm, MIMU provided in the MIMU local frame $(\mathcal{M})$, at each time $t$, the acceleration, the angular velocity and the orientation relative to the earth reference frame $(\mathcal{E})$ (north,

122 acceleration in the earth frame $\mathcal{E}$ is: $\boldsymbol{a}_{\mathcal{E}, t}=\left[\begin{array}{lll}a_{t}^{n} & a_{t}^{w} & a_{t}^{u}\end{array}\right]$.

123 A technical calibration as proposed by (Bouvier et al., 2015) was performed to register the local frame

124 of the $\mathrm{MIMU}(\mathcal{M})$ with the anatomical axes of the trunk (i.e. proximal-distal (PD), medio-lateral (ML),

125 antero-posterior (AP) axes). Thus, the linear acceleration was deduced in the trunk reference frame 
$126(\mathcal{T})$ as $\boldsymbol{a}_{\mathcal{T}, \boldsymbol{t}}=\left[\begin{array}{lll}a_{t}^{P D} & a_{t}^{M L} & a_{t}^{A P}\end{array}\right]$. By the same procedure, the angular velocity of the trunk was

127 deduced in the torso frame as $\omega_{\mathcal{T}, t}=\left[\begin{array}{lll}\omega_{t}^{P D} & \omega_{t}^{M L} & \omega_{t}^{A P}\end{array}\right]$.

128 The inclination angle of the torso $\theta_{t}$ was computed as the angle between the axis of the torso and the

129 vertical axis. Then, the STS beginning time $t_{b}$, the STS end time $t_{f}$, the velocity of the torso center of

130 mass $\left(\boldsymbol{V} \boldsymbol{G}_{\mathcal{T}, t}\right)$ and the kinetic energy $\left(\boldsymbol{E} \boldsymbol{K}_{\boldsymbol{t}}\right)$ of the torso were computed (Lepetit et al., 2018).

131 In the STS time window, for each subject, 15 parameters were computed as the average value of all

132 trials as follows:

- TD: the STS task duration such as TD $=t_{f}-t_{b}$;

134

- mAcc and maxAcc: the mean and maximal values of the norm of $\boldsymbol{a}_{\varepsilon, t}$;

135

- $\quad$ mAz and maxAz: the mean and maximal values of the absolute value of $\boldsymbol{a}_{\mathcal{\varepsilon}, \boldsymbol{t}}$ along the vertical

136 axis $\left|a_{t}^{u}\right|$

137

138

- mAxy and maxAxy: the mean and maximal values of the norm of $\boldsymbol{a}_{\varepsilon, t}$ in the horizontal plane lateral sway (W. Janssen et al., 2008) (AUCml $\left.=\int_{t_{b}}^{t_{f}}\left|a_{t}^{M L}\right| d t\right)$;

- $\quad$ mVG and maxVG: the mean and maximal values of the norm of the torso COM velocity $\boldsymbol{V} \boldsymbol{G}_{\mathcal{T}, t}$;

- $\quad$ mEK and maxEK: the mean and maximal values of the norm of the torso kinetic energy $\boldsymbol{E} \boldsymbol{K}_{\boldsymbol{t}}$; velocity $\omega_{\mathcal{T}, t}$ 

$\left.\max \left(\left|\theta_{t}\right|\right)\right)$

147 To investigate the age effect on each parameter, a Mann-Whitney U-test was realized between the parameters of HY and HS groups. Likewise, the influence of frailty was analyzed with a Mann-Whitney U-test realized between HS and frail groups. The significance level was set to 0.01 for all comparisons.

To assess the discrimination performance of each parameter between $\mathrm{HY}$ and $\mathrm{HS}$, the area under the curve (AUC) of a receiver operating characteristic (ROC) was computed (Zweig \& Campbell, 1993).

157 The aim of the AgingScore is to reduce the $k$-length vector to a scalar based on an iterative principal component analysis (PCA) procedure as follows. First, from the $k$-length vectors of the HS and HY subjects, an $a$-length sub-vectors of the $a$ most discriminative parameters according to the PCA $(1 \leq$ $a \leq k$ ) were extracted (Jackson, 1991). At this stage, each subject is now characterized by an $a$-length vector. Secondly, the $a$-length vectors of HY and HS subjects were randomly divided into equal training 
HY_S and HS_S subjects and was defined as the temporary aging score named AgingScore-tmp.. At this

Finally, the value $a$ was chosen in order to maximize the classification performance $A U C$ - $a$. In addition, the $a$-length vector associated to the AgingScore identified the parameters related with age.

The same procedure was implemented to assess the FrailtyScore based on the $f$-length vectors with FrailtyScore identified the parameters related to frailty. 
183

184

185

186

187

188

189

190

191

192

193

194

195

196

197

198

199

200

deduce a s-length vector with $\max (a, f) \leq s \leq a+f$. The vector of the $s$ unique parameters was normalized by the mean values of the HY group. For each group, the mean and standard deviation values of each parameter were displayed in a radar plot. For each subject, the STS strategy was quantified by computing the circularity ratio $\left(\frac{\text { perimeter }^{2}}{4 \pi . \text { area }^{2}}\right)$ of the polygon in the radar plot. The significance of the evolution of the circularity ratio between groups was quantified with a MannWhitney U-test. Data are presented as mean and standard deviation. The significance level was set to 0.01

\section{RESULTS}

The mean and standard deviation for each of the 15 parameters for each group are presented in table

2. The p-values of Mann-Whitney U-tests are also given. A significant difference between HY and HS subjects has been found for maxVG, maxOmega, maxAcc, maxAz, while between HS and FS subjects, significant differences were highlighted for maxAxy, mVG, maxVG, mOmega, TD, Incl, mAcc, mAz, mAxy, AUCml, mEK and maxEK.

By the value of AUC of the ROC analysis, it was demonstrated that maxAcc was the most discriminative for $\mathrm{HY}$ and $\mathrm{HS}$ groups (AUC=0.763), and $\mathrm{mVG}$ was the most discriminative for HS and FS groups (AUC=0.972).

The $a$-vector of parameters which maximized the AgingScore discrimination performance was [maxAcc, maxAz, maxAxy, maxVG, maxOmega] with a=5. For the FrailtyScore, the $f$-vector of 

AUCml] $(f=7$ ) (table 2).

The fraction of variance explained by the first principal component was $77.48 \pm 2.80 \%$ for PCA with HY and HS groups and $74.94 \pm 2.24 \%$ for PCA with HS and FS groups. The average ROC curve and AUC for both classifications with AgingScore and FrailtyScore are displayed in figure 1.

Insert figure 1

Insert figure 2

The quantification of the STS postural transition with a single MIMU fixed on the trunk enabled the classification of the subjects according to two different scores. Moreover, the present study has evidenced that the analysis based on 12 parameters was able to quantify the strategy of the STS 
motion. The influence of age and frailty on the STS movement through several parameters was

220 demonstrated. The results also validated that the STS motion strategy was significantly modified for

221 few frail subjects.

222 Classically, the task duration (TD) is the only parameter analysed during single STS transition. The mean

223 TD values for healthy subjects were between 1.57s and 2.42s (Cerrito et al., 2015; Galli et al., 2008;

showed that TD increases with frailty (Ganea et al., 2011; Millor et al., 2013; R. C. Van Lummel et al., 2013). However, there is no consensus for the influence of age. Studies showed that the subject's age may influence (R. C. Van Lummel et al., 2013) or not (Hurley, 2013) this parameter during the STS motion. This could be explained by the different methodological approaches used to determinate $t_{b}$ and $t_{f}$. Hurley used a marker-based motion capture device in his study which is known to be more reliable than magneto-inertial units used by (R. C. Van Lummel et al., 2013). heterogeneities according to age and frailty. For instance, the maximal value of the trunk CoM velocity (maxVG) was the only parameter which was significantly influenced by age and frailty. The inclination angle (Incl) did not evolve significantly with the age but raised with frailty. Although the mean value increased for FS subjects, the range of values was wider (standard deviation $=20.70^{\circ}$ ). However, the inclination angle was similar between HY and HS subjects and in agreement with a previous study (Hurley, 2013). Also, AUCml which is linked to the acceleration and TD did not evolve between HY and 
the STS performance evaluate by single parameter would not enough be accurate, and consequently,

240 the use of a composite parameter, i.e. a score, as an image of multidimensional parameters, is more

relevant (W. G. M. Janssen et al., 2008).

242 In the present study, a multifactorial analyze of several parameters was reduced into a unique quantitative score using the first principal component of a PCA. The classification performance of these scores were quantified with a ROC analysis. In both cases, the AUC which represents the classification performance of the scores was better than with any other single parameter (figure 1 and table 2). The FrailtyScore enabled a reliable classification (meanAUC $>0.98$, figure 1 ). This result was improved in comparison to previous studies which generally used only one parameter such as TD (Millor et al., 2014). The AgingScore enabled to classify HS and HY subjects (meanAUC $>0.77$, figure 1). Van Lummel (R. Van Lummel, 2017) proposed a score to evaluate the 5 times repeated STS. Their method was based on an exploratory factor analysis of 24 parameters of three different types: durations, kinematics and coefficients of variation. However, the discrimination power between young and old individuals was not documented because they did not include young subjects in their study. showed that except for maxEK, all the maximal values of the other parameters decreased significantly with age. These results could be explained by a reduction of muscles and tendons capacities. Indeed, the relationship between muscle strength and STS performance was already demonstrated (Bohannon et al., 2010). On other hand, the circularity ratio analysis demonstrated that the STS strategy is not significantly influenced with age $(p$-value $=0.221)$ for the healthy subjects. This result agreed with a 
previous study which highlighted quantitative reduction but similar qualitative kinematic and kinetic parameters between HY and HS subjects (Hurley, 2013; Marin et al., 1999; Steffen et al., 2013). On the contrary, frailty influences significantly the STS strategy ( $p$-value $<0.01$ ). We found that all the mean-based parameters (mVG, mAcc, mAz, mAxy, mEK), max EK and maxVG decreased significantly for FS subjects as compared with HS and HY groups. These observations could be a marker of frailness for further longitudinal investigation. However, this study also has some limitations. First, frail subjects were older than healthy seniors. Secondly, with our methodology, the computed variables often required the determination of $t_{b}$ and $t_{f}$. In the literature, numerous methods to detect movement are proposed with MIMU data without consensus (Cerrito et al., 2015; Millor et al., 2013). In this study, the motion detection algorithm was based on a threshold of the orientation quaternions and the vertical acceleration (Lepetit et al., 2018). Moreover, the parameters based on maximum values were often more dispersed than those based on mean value (table 2). Indeed, they focused on only one specific moment and consequently, they were more subject to sensor errors. Finally, the muscle strength and activation were not evaluated, and it may be useful to add this information. premature functional deficit with a single MIMU during the STS transition. This setup is appropriate for clinical routines and may help clinicians to detect subject with abnormal functional capacities and monitor rehabilitation enhancements. 
The authors have no conflicts of interest to report related to this study.

\section{ACKNOWLEDGEMENTS}

The research leading to these results has received funding from "Agence National de la Recherche"

ANR-11-IDEX-0004-02 under Idex "Sorbonne Universités" and PhD funding of ENS Cachan.

\section{REFERENCES}

Alexander, N. B., Schultz, A. B., \& Warwick, D. N. , 1991. Rising From a Chair: Effects of Age and Functional Ability on Performance Biomechanics. Journal of Gerontology: MEDICAL SCIENCES, 46(3), 91-98.

Beauchet, O., Fantino, B., Allali, G., Muir, S. W., Montero-Odasso, M., \& Annweiler, C. , 2011. Timed Up and Go test and risk of falls in older adults: a systematic review. The Journal of Nutrition, Health \& Aging, 15(10), 933-8.

Bohannon, R. W., Bubela, D. J., Magasi, S. R., Wang, Y.-C., \& Gershon, R. C. , 2010. Sit-to-stand test: Performance and determinants across the age-span. Isokinetics and Exercise Science, 18(4), 235240.

Bouvier, B., Duprey, S., Claudon, L., Dumas, R., \& Savescu, A. , 2015. Upper Limb Kinematics Using Inertial and Magnetic Sensors: Comparison of Sensor-to-Segment Calibrations. Sensors, 15(8), 18813-18833.

Cerrito, A., Bichsel, L., Radlinger, L., \& Schmid, S. , 2015. Reliability and validity of a smartphone-based application for the quantification of the sit-to-stand movement in healthy seniors. Gait \& Posture, 41(2), 409-413.

Edward Jackson, J. , 1991. A user's guide to principal components. Wiley-Interscience Paperback Series.

Fried, L. P., Tangen, C. M., Walston, J., Newman, A. B., Hirsch, C., Gottdiener, J., ... McBurnie, M. A. , 2001. Frailty in Older Adults: Evidence for a Phenotype. The Journals of Gerontology Series A: Biological Sciences and Medical Sciences, 56(3), M146-M157.

Galli, M., Cimolin, V., Crivellini, M., \& Campanini, I. , 2008. Quantitative analysis of sit to stand movement: Experimental set-up definition and application to healthy and hemiplegic adults. Gait 
Ganea, R., Paraschiv-lonescu, A., Büla, C., Rochat, S., \& Aminian, K. , 2011. Multi-parametric evaluation of sit-to-stand and stand-to-sit transitions in elderly people. Medical Engineering \& Physics, 33(9), 1086-93.

Grimm, B., \& Bolink, S. , 2016. Evaluating physical function and activity in the elderly patient using wearable motion sensors. EFORT Open Reviews, 1(5), 112-120.

Howcroft, J., Kofman, J., \& Lemaire, E. D. , 2013. Review of fall risk assessment in geriatric populations using inertial sensors. Journal of Neuroengineering and Rehabilitation, 10(1), 91.

Hurley, S. T. , 2013. Sit-to-stand transfer mechanics: the effect of age and lifting-seat device design. Dalhousie University, Halifax, Nova Scotia.

Jackson J. E. ,1991, A Use's Guide to Principal Components, Book Series:Wiley Series in Probability and Statistics, John Wiley \& Sons

Janssen, W. G. M., Bussmann, J. B. J., Horemans, H. L. D., \& Stam, H. J. , 2008. Validity of accelerometry in assessing the duration of the sit-to-stand movement. Medical \& Biological Engineering \& Computing, 46(9), 879-887.

Janssen, W., Kulcu, D. G., Horemans, H., Stam, H. J., \& Bussmann, J. , 2008. Sensitivity of Accelerometry to Assess Balance Control During Sit-to-Stand Movement. IEEE Transactions on Neural Systems and Rehabilitation Engineering, 16(5), 479-484.

Jolliffe, I. T. , 2002. Principal Component Analysis, Second Edition. (P. Bickel, P. Diggle, S. Fienberg, K. Krickeberg, I. Olkin, N. Wermuth, \& S. Zeger, Eds.). Springer.

Lepetit, K., Ben Mansour, K., Boudaoud, S., Kinugawa-Bourron, K., \& Marin, F. , 2018. Evaluation of the kinetic energy of the torso by magneto-inertial measurement unit during the sit-to-stand movement. Journal of Biomechanics, 67, 172-176.

Marin, F., Allain, J., Diop, A., Maurel, N., Simondi, M., \& Lavaste, F. , 1999. On the estimation of knee joint kinematics. Human Movement Science, 18(5), 613-626.

Mijnarends, D. M., Meijers, J. M. M., Halfens, R. J. G., ter Borg, S., Luiking, Y. C., Verlaan, S., ... Schols, J. M. G. A. , 2013. Validity and Reliability of Tools to Measure Muscle Mass, Strength, and Physical Performance in Community-Dwelling Older People: A Systematic Review. Journal of the American Medical Directors Association, 14(3), 170-178.

Millington, P. J., Myklebust, B. M., \& Shambes, G. M. , 1992. Biomechanical Analysis of the Sit-to-Stand Motion in Elderly Persons. Arch Phys Med Rehabilitation, 73, 609-617.

Millor, N., Lecumberri, P., Gomez, M., Martinez-Ramirez, A., \& Izquierdo, M. , 2014. Kinematic Parameters to Evaluate Functional Performance of Sit-to-Stand and Stand-to-Sit Transitions Using Motion Sensor Devices: A Systematic Review. IEEE Transactions on Neural Systems and Rehabilitation Engineering, 22(5), 926-936.

Millor, N., Lecumberri, P., Gómez, M., Martínez-Ramírez, A., \& Izquierdo, M. , 2013. An evaluation of the 30-s chair stand test in older adults: frailty detection based on kinematic parameters from a single inertial unit. Journal of Neuroengineering and Rehabilitation, 10, 86.

Moufawad el Achkar, C., Lenbole-Hoskovec, C., Paraschiv-lonescu, A., Major, K., Büla, C., \& Aminian, K. , 2018. Classification and characterization of postural transitions using instrumented shoes. Medical \& Biological Engineering \& Computing, 1-10.

Mugueta-Aguinaga, I., \& Garcia-Zapirain, B. , 2017. Is Technology Present in Frailty? Technology a Back- 
up Tool for Dealing with Frailty in the Elderly: A Systematic Review. Aging and Disease, 8(2), 176195.

Nikas, J. B., \& Low, W. C. , 2011. ROC-supervised principal component analysis in connection with the diagnosis of diseases. American Journal of Translational Research, 3(2), 180-96.

Nuzik, S., Lamb, R., VanSant, A., \& Hirt, S. , 1986. Sit-to-stand movement pattern. A kinematic study. Physical Therapy, 66(11), 1708-13.

Rockwood, K., Song, X., MacKnight, C., Bergman, H., Hogan, D. B., McDowell, I., \& Mitnitski, A. , 2005. A global clinical measure of fitness and frailty in elderly people. CMAJ : Canadian Medical Association Journal = Journal de l'Association Medicale Canadienne, 173(5), 489-95.

Sabatini, A. M. , 2011. Estimating three-dimensional orientation of human body parts by inertial/magnetic sensing. Sensors, 11(2), 1489-1525.

Steffen, D., Bleser, G., Weber, M., Stricker, D., Fradet, L., \& Marin, F. , 2013. A personalized exercise trainer for elderly. Journal of Ambient Intelligence and Smart Environments, 5(6), 24-31.

Sun, R., \& Sosnoff, J. J. , 2018. Novel sensing technology in fall risk assessment in older adults: a systematic review. BMC Geriatrics, 18(1), 14.

Van Lummel, R. , 2017. Assessing Sit-to-Stand for Clinical Use. Retrieved from https://www.mcroberts.nl/wp-content/uploads/2017/03/complete-dissertation.pdf

Van Lummel, R. C., Ainsworth, E., Lindemann, U., Zijlstra, W., Chiari, L., Van Campen, P., \& Hausdorff, J. M. , 2013. Automated approach for quantifying the repeated sit-to-stand using one body fixed sensor in young and older adults. Gait \& Posture, 38(1), 153-156.

Zijlstra, W., Bisseling, R. W., Schlumbohm, S., \& Baldus, H. , 2010. A body-fixed-sensor-based analysis of power during sit-to-stand movements. Gait \& Posture, 31(2), 272-8.

Zweig, M. H., \& Campbell, G. , 1993. Receiver-operating characteristic (ROC) plots: a fundamental evaluation tool in clinical medicine. Clinical Chemistry, 39(4), 561-77. Retrieved from http://www.ncbi.nlm.nih.gov/pubmed/8472349 


\section{Tables}

\begin{tabular}{|c|c|c|c|c|c|c|}
\cline { 2 - 7 } \multicolumn{1}{c|}{} & $\mathrm{n} \uparrow$ & $\mathrm{n} \circlearrowleft$ & Age (years) & Height $(\mathbf{c m})$ & Weight $(\mathbf{k g})$ & BMI (kg/m $\mathbf{2})$ \\
\hline HY subjects & 4 & 20 & $25(3)$ & $178(9.5)$ & $72.1(11.7)$ & $22.8(3.1)$ \\
\hline HS subjects & 5 & 34 & $70(4)$ & $174(8.3)$ & $79.4(14.2)$ & $26.1(4.1)$ \\
\hline FS subjects & 6 & 5 & $87(6)$ & $161(6.0)$ & $61.0(11.2)$ & $23.6(4.9)$ \\
\hline \multicolumn{7}{|c|}{ Table 1: Subjects' characteristics: mean value (standard deviation) } \\
\end{tabular}




\begin{tabular}{|l|c|c|c|c|c|c|}
\hline \multicolumn{1}{|c|}{ Parameter } & $\begin{array}{c}\text { Healthy young } \\
\text { subjects (HY) }\end{array}$ & $\begin{array}{c}\text { Healthy seniors } \\
\text { subjects (HS) }\end{array}$ & $\begin{array}{c}\text { Frail subjects } \\
\text { (FS) }\end{array}$ & $\begin{array}{c}\text { p-value } \\
\text { (HY,HS) }\end{array}$ & $\begin{array}{c}\text { ROC AUC } \\
\text { (HY,HS) }\end{array}$ & $\begin{array}{c}\text { R-value } \\
\text { (HS,FS) }\end{array}$ \\
\hline mVG (m/s) FS)
\end{tabular}

Table 2: Mean ( standard deviation) for the parameters evaluating during the sit-to-stand. The Mann-Whitney $p$-values and ROC AUC values were assessed between healthy young subjects (HY) and healthy senior subjects (HS) and between HS subjects and frail subjects (FS). 


\section{Figure Captions}

Figure 1: Average ROC curves (dark lines) and standard deviation limits (shaded areas) quantifying the classification performance of the PCA-based scores between HY and HS subjects (AgingScore, left) and between HS and FS subjects (FrailtyScore, right). The mean AUC values and their standard deviations are given for each curve.

Figure 2: Medians (dark lines), $1^{\text {st }}$ and $3^{\text {rd }}$ quartiles (lower and upper limits of shaded areas) of retained parameters for all groups presented in a radar plot. The data were normalized according to the median values of HY group (thus, red dark line is the unit circle).

Figure 3: The circularity ratio of each group is presented in a boxplot. The evolution between groups was investigated with Mann-Whitney U-tests. The evolution was significative between HY and FS groups and between HS and FS groups. 

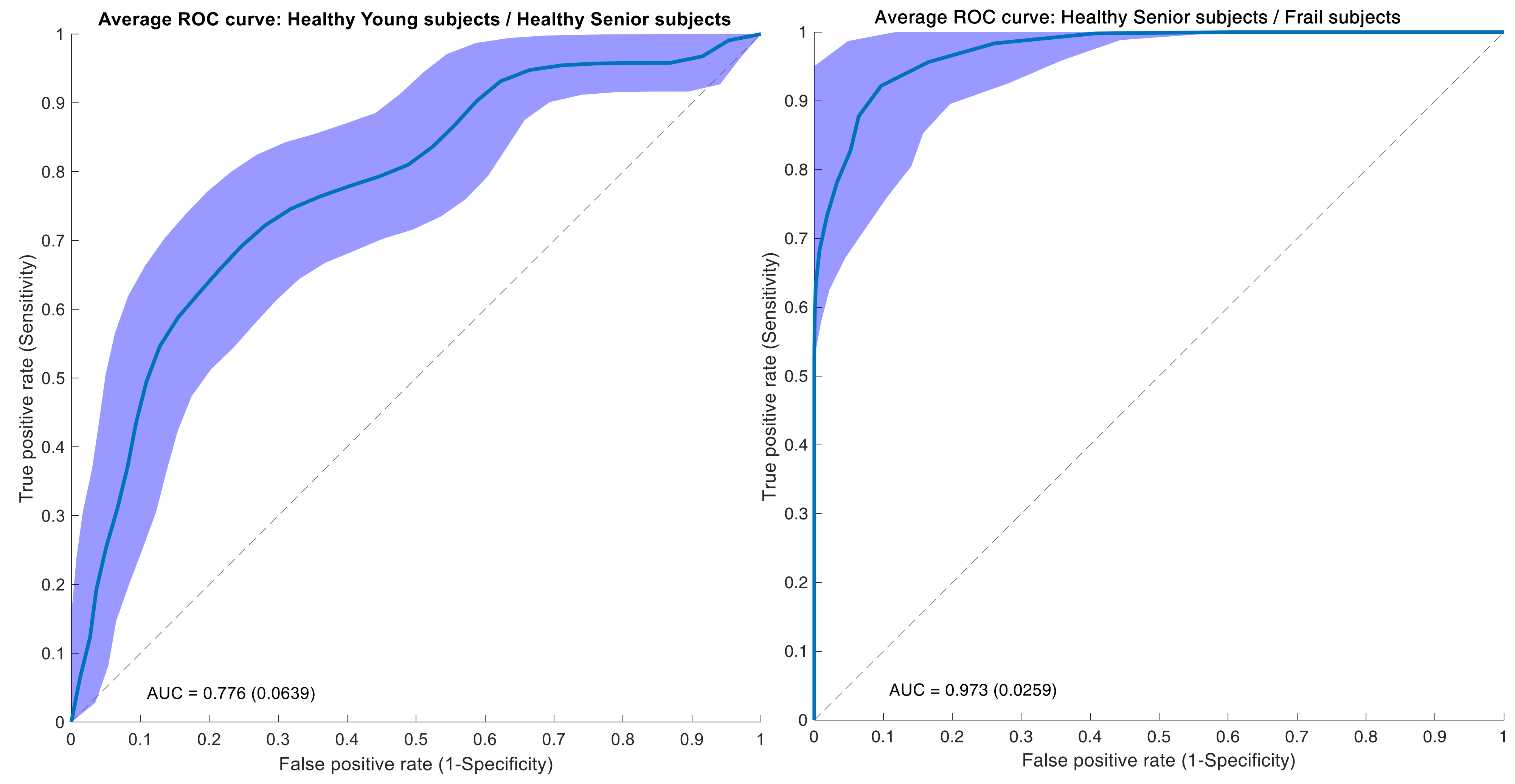


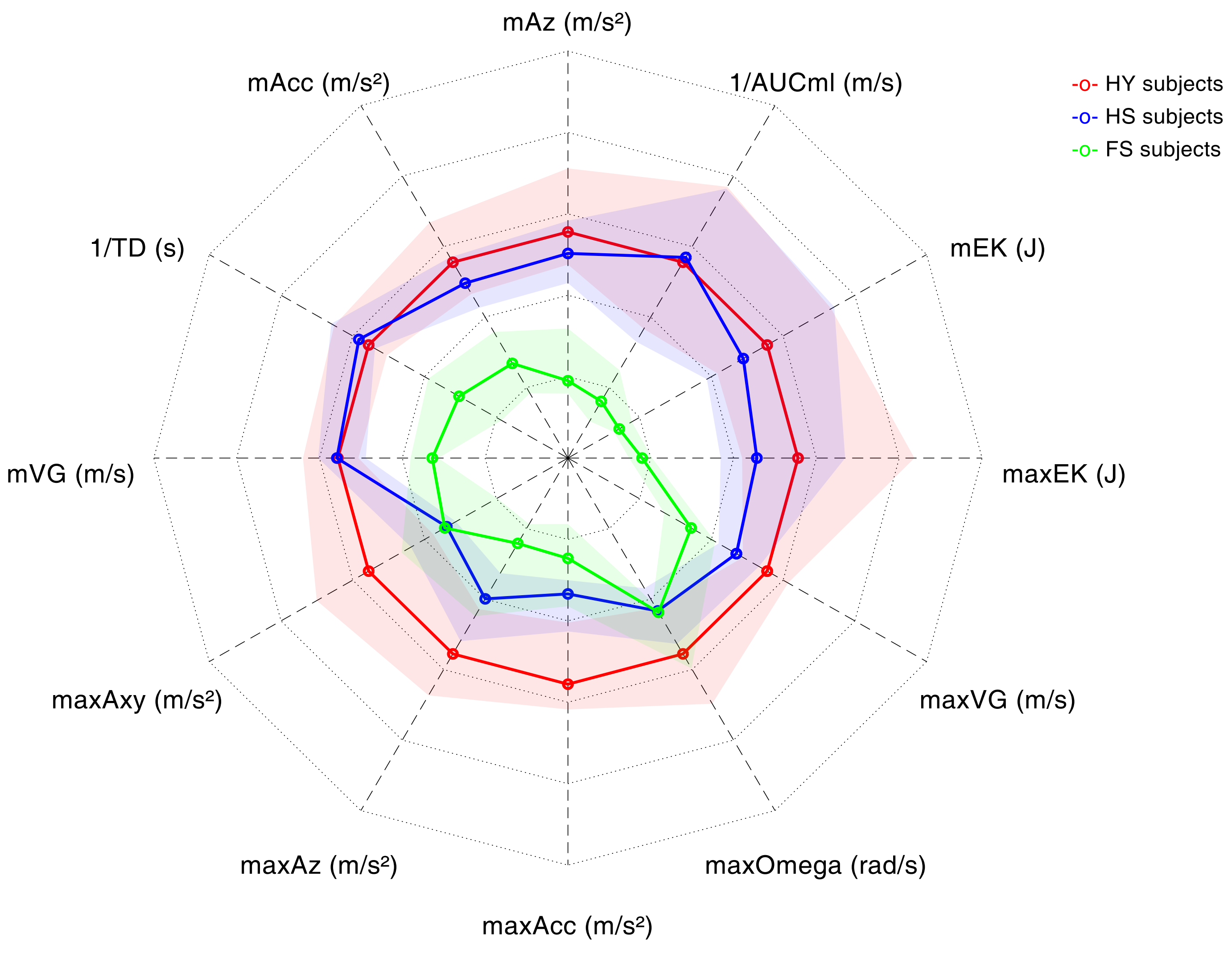




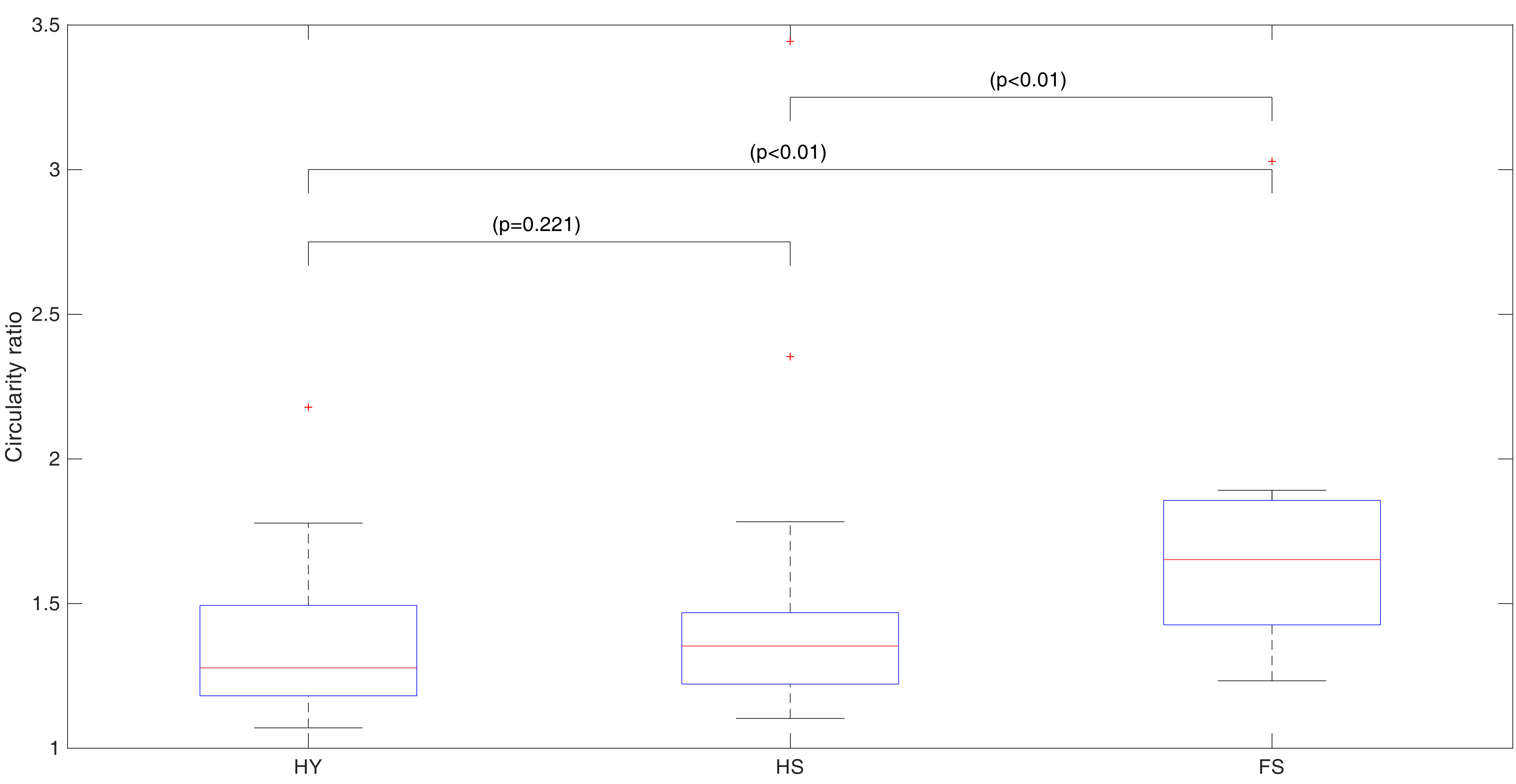

